\title{
BMJ Open Excess burden of non-communicable disease years of life lost from heat in rural Burkina Faso: a time series analysis of the years $2000-2010$
}

\author{
Aditi Bunker, ${ }^{1,2}$ Maquins Odhiambo Sewe, ${ }^{3}$ Ali Sié, ${ }^{4}$ Joacim Rocklöv, ${ }^{3}$ \\ Rainer Sauerborn ${ }^{2}$
}

To cite: Bunker A, Sewe M0, Sié $A$, et al. Excess burden of non-communicable disease years of life lost from heat in rural Burkina Faso: a time series analysis of the years 2000-2010. BMJ Open 2017;7:e018068. doi:10.1136/ bmjopen-2017-018068

\section{- Prepublication history and} additional material for this paper are available online. To view these files, please visit the journal (http://dx.doi.org/10. 1136/bmjopen-2017-018068)

Received 5 June 2017 Revised 15 September 2017 Accepted 28 September 2017

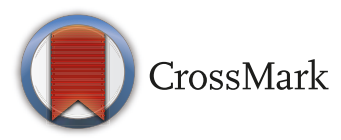

${ }^{1}$ Network Aging Research, Heidelberg University, Heidelberg, Germany ${ }^{2}$ Institute of Public Health, University of Heidelberg, Heidelberg, Germany ${ }^{3}$ Epidemiology and Global Health, Department of Public Health and Clinical Medicine, Umeå University, Umeå, Sweden ${ }^{4}$ Centre de Recherche en Santé de Nouna, Nouna, Burkina Faso

Correspondence to

Aditi Bunker;

aditi.bunker@uni-heidelberg.de

\section{ABSTRACT}

Objectives Investigate the association of heat exposure on years of life lost (YLL) from non-communicable diseases (NCD) in Nouna, Burkina Faso, between 2000 and 2010.

Design Daily time series regression analysis using distributed lag non-linear models, assuming a quasiPoisson distribution of YLL.

Setting Nouna Health and Demographic Surveillance System, Kossi Province, Rural Burkina Faso.

Participants 18367 NCD-YLL corresponding to 790 NCD deaths recorded in the Nouna Health and Demographic Surveillance Site register over 11 years.

Main outcome measure Excess mean daily NCD-YLL were generated from the relative risk of maximum daily temperature on NCD-YLL, including effects delayed up to 14 days.

Results Daily average NCD-YLL were 4.6, 2.4 and 2.1 person-years for all ages, men and women, respectively. Moderate 4-day cumulative rise in maximum temperature from $36.4^{\circ} \mathrm{C}$ (50th percentile) to $41.4^{\circ} \mathrm{C}$ (90th percentile) resulted in 4.44 (95\% $\mathrm{Cl} 0.24$ to 12.28 ) excess daily NCDYLL for all ages, rising to 7.39 (95\% $\mathrm{Cl} 0.32$ to 24.62$)$ at extreme temperature $\left(42.8^{\circ} \mathrm{C}\right.$; 99 th percentile). The strongest health effects manifested on the day of heat exposure (lag 0), where 0.81 (95\% Cl 0.13 to 1.59) excess mean NCD-YLL occurred daily at $41.7^{\circ} \mathrm{C}$ compared with $36.4^{\circ} \mathrm{C}$, diminishing in statistical significance after 4 days. At lag 0 , daily excess mean NCD-YLL were higher for men, 0.58 (95\% Cl 0.11 to 1.15) compared with women, 0.15 (95\% $\mathrm{Cl}-0.25$ to 9.63 ) at $41.7^{\circ} \mathrm{C}$ vs $36.4^{\circ} \mathrm{C}$.

Conclusion Premature death from NCD was elevated significantly with moderate and extreme heat exposure. These findings have important implications for developing adaptation and mitigation strategies to reduce ambient heat exposure and preventive measures for limiting NCD in Africa.

\section{INTRODUCTION}

As the global average temperature rises, epidemiological evidence on the temperature-health association in neglected African populations is needed to develop appropriate interventions. Surface temperature
Strengths and limitations of this study

- This study investigated the relationship between two defining public health issues affecting Sub-Saharan Africa: increasing ambient heat from climate change and the rising prevalence of non-communicable disease.

- Eleven years of high-quality health and demographic data from rural Africa was exploited for analysis.

- Only premature death was quantified as the outcome because long-term morbidity data were unavailable.

- Temperature data from a weather station located $53 \mathrm{~km}$ from the study location were used as a proxy for individual level temperature exposure.

over West Africa and the Sahel increased by $0.5^{\circ} \mathrm{C}-0.8^{\circ} \mathrm{C}$ between 1970 and 2010 and at a faster pace in the most recent 20 years. ${ }^{1}$ Analysis of longitudinal data from 12 Health and Demographic Surveillance Sites (HDSS), which include the Nouna HDSS in Burkina Faso, forecasts that the mean temperature in Africa will exceed the 1900-2000 decadal average by 2100 under all climate change scenarios. ${ }^{2}$ In a study applying six climate model-future scenarios across six HDSS sites, the most conservative combination, rapid economic growth and balanced energy sources, resulted in a $0.5^{\circ} \mathrm{C}-1^{\circ} \mathrm{C}$ temperature increase by 2100 , whereas most combinations projected a $2^{\circ} \mathrm{C}-3^{\circ} \mathrm{C}$ temperature rise in the same period. ${ }^{2}$ Prolonged exposure to high ambient temperature in the subsistence farming community of Nouna, and low adaptive capacity makes this community particularly vulnerable to the effects of temperature increase.

Non-communicable disease (NCD) causes substantial economic drain to society by adversely affecting four pillars of economic growth: labour supply, productivity, investments and education. Projections from 2006 
indicated that if no action was taken to reduce the risk of NCD in 23 low-income and middle-income countries, US $\$ 83$ billion would be lost over the subsequent decade to the impact of heart disease, stroke and diabetes. ${ }^{3}$ As life expectancy increases in Burkina Faso, people will have more time to develop chronic and degenerative disorders; NCD will therefore contribute increasingly to population mortality. In 2014, NCD accounted for $32 \%$ of all deaths in Burkina Faso. The main contributors were cardiovascular disease, cancer, chronic respiratory disease and diabetes. ${ }^{4}$ In 2011, Friel et al presented a review exposing the link between climate change and a wide range of $\mathrm{NCD}$, and argued that more frequent and intense heat extremes could exacerbate cardiovascular and respiratory health outcomes. ${ }^{5}$

Previous studies have explored the impact of extreme events such as heatwaves or cold waves ${ }^{67}$ on health, which are anticipated to increase in frequency and magnitude with climate change. ${ }^{8}$ A recent multicity study, however, found that milder non-optimal temperature rather than extreme temperature was responsible for most of the temperature-related mortality burden (defined as below the 2.5 th percentile and above the 97.5 th percentile). ${ }^{9}$ Unfortunately, no African studies were included by Gasparrini et al. ${ }^{9}$ Heat (and cold) waves are defined by magnitude and duration; for example, 2 or more consecutive days exceeding the 98th-99th (or 1st-2nd) percentiles of the temperature range. Excess risks are a comparison of heatwave periods with non-heatwave periods in previous years. Our study investigates the health risks of moderate to extreme heat, where extreme temperature is defined as below the 5 th percentile and above the 95 th percentile of maximum temperature.

Epidemiological studies on the temperature-health association in African populations have primarily measured daily deaths as the outcome. ${ }^{10}{ }^{11}$ Rather than the number of deaths, we used years of life lost (YLL), a global burden of disease (GBD) outcome metric for ascertaining premature death. YLL are an aggregate of life expectancy and death counts that gives the absolute value of YLL from a certain exposure, rather than a relative risk (RR). In the only previous study set in Africa investigating the temperature-YLL association, Egondi et al found no heat effects on all-cause YLL in the East African highlands of Nairobi, Kenya. A reduction in temperature $\left(21^{\circ} \mathrm{C}\right.$ compared with $\left.26^{\circ} \mathrm{C}\right)$, however, resulted in 27.4 excess all-cause YLL per day (95\% CI 2.7 to 52.0). ${ }^{12}$ The current article addresses Africa's dual challenge of coping with rising temperatures from climate change and increasing prevalence of NCD. The association between temperature and other health outcomes in Nouna, including infectious disease, will be the subject of future work. The paucity of population-based studies set in Africa focused on the impact of temperature on NCD health outcomes suggests further studies are required. Our study addresses this research gap by investigating the impact of 11 years of heat exposure on YLL from NCD in the Nouna HDSS.

\section{METHODS}

\section{Data collection}

Health outcomes data were obtained from the HDSS, Centre de Recherche en Santé de Nouna, Burkina Faso. ${ }^{13}$ All registered deaths between 1 January 2000 and 31 December 2010 were included. Vital statistics for each resident included a unique identifying number (ID), date of birth, date of immigration into the HDSS, date of death, date of emigration from the HDSS and gender. Raw mortality data comprised a unique ID number for each death event, date of birth, date of death, sex, cause of death coded as an ICD-10 (International Classification of Diseases) code and an accompanying cause of death in French. Cause of death was established by verbal autopsy. ${ }^{14}$ Age of death was calculated as the difference between the date of death and birth. We applied the GBD cause-specific categories and ICD-10 codes to define NCD as an aggregate of: malignant neoplasms (C00-C97), other neoplasms (D00-D48), diabetes mellitus (E10-E14), endocrine disorders (D55-D64; minus-D64.9, D65-D89, E03-E07, E15-16, E20-E34, E65-E88), neuropsychiatric conditions (F01-F99, G06-G98), sense organ diseases (H00-H61, H68-H93), cardiovascular diseases (I00-I99), respiratory diseases (J30-J98), digestive diseases (K00K92), genitourinary diseases (N00-N64, N75-N98), skin diseases (L00-L98), musculoskeletal diseases (M00-M99) and congenital anomalies (Q00-Q99). ${ }^{15}$

\section{Computation of daily YLL}

Different resolutions of life tables can be used to calculate YLL, that is, global, country-level or local life expectancy depending on the purpose of the study. In 1990, the GBD approach calculated YLL relative to the life expectancy of Japanese men and women, the highest for any societal group. ${ }^{16}$ Weights for age and time preference can additionally be applied to reduce the contribution of death before adulthood. ${ }^{17}$ For the GBD 2010 study, a reference standard of 86 years at birth was used for both men and women and YLL were calculated using a life table based on the lowest observed mortality in each age group in countries with more than 5 million inhabitants. ${ }^{18}$ This study used local rather than global life tables, as done in similar studies, ${ }^{19-21}$ to present realistic potential losses or gains in life years for the Nouna population grounded in real data (rather than modelled data), which is more meaningful for local decision-makers. The cause of death and demographic data from the Nouna HDSS were used to build life tables for the Nouna population. The use of global life expectancy would likely produce very large YLL for populations with low life expectancy such as in Burkina Faso. Furthermore, global life expectancy is likely to be more useful when comparing YLL between two countries, which was not the aim of this study.

We used the Nouna HDSS vital events and mortality data from 2000 to 2010 to produce age-specific death rates. We generated gender-specific life tables to account for varying life expectancies between men and women (details in online supplementary tables 1 and 2). Mean 
additional survival time, averaged between 2000 and 2010, was calculated for each age band to account for the changing population profile over this time. Abridged life tables were created in 5-year increments, producing stable life expectancy estimates for a relatively small population (approximately 90000 inhabitants in $2012^{10}$ ). The $0-1$ and $1-5$ age groups were, however, separated. Combining these ages would mask the lower remaining life expectancy for the $0-1$ age group relative to the 1-5 age group, a consequence of high infant mortality. For each NCD death, YLL were calculated by matching age and sex with the relevant life table. Daily YLL were an aggregate of individual YLL on the respective day calculated as:

(A) individual YLL at time of death,

$$
\mathrm{YLL}_{\text {individual }(i)}=\mathrm{LE}_{\text {remaining-Age }} \text { death }
$$

(B) total daily YLL,

$$
Y L L_{d a i l y}=\sum_{i=1}^{n} Y L L_{i}
$$

where:

$i$ is the $i$ th individual,

$L E_{\text {remaining }}$ is the conditional life expectancy,

Age $_{\text {death }}$ is the age at death,

$n$ is the number of deaths occurring on a given day.

We stratified NCD-YLL by sex to assess if gender differences existed.

\section{Temperature data}

Because temperature data for Nouna were not sufficiently complete for analysis, we obtained hourly mean (t-mean), maximum temperature (t-max) and minimum temperature (t-min) data from the National Climatic Data Centre for the Dédougou weather station $\left(12.4^{\circ} \mathrm{N}\right.$, $3.4^{\circ} \mathrm{W}$ ) from 1 January 2000 to 31 December 2010 (4071 days). Pearson's correlation analysis was performed to compare maximum temperature between a local Nouna weather station (coordinates $12.7^{\circ} \mathrm{N}, 3.9^{\circ} \mathrm{W}$ ) and the
Dédougou weather station (located $53 \mathrm{~km}$ from Nouna). Over the study period of 4071 days, 2432 days (59\%) of maximum temperature from Nouna were available for comparison. The very strong correlation coefficient of 0.93 (95\% CI 0.92 to 0.94$), \mathrm{p}<2.2 \mathrm{e}-16$, indicated there was little variability between the two sites, validating our use of Dédougou maximum temperature for Nouna. Hourly Dédougou data were averaged to give a daily temperature. The raw time series consisted of $25 \%$ missing t-mean, $14 \%$ t-max and $17 \%$ t-min. We created an imputation algorithm by averaging 15 consecutive days of temperature either side of a missing temperature value to create a 30 -day moving average. The Time Indexes and Time Indexed Series (tis) package V.1.30 was applied in $\mathrm{R}$ software to impute missing temperature values.

\section{Statistical modelling}

We applied time series quasi-Poisson regression analysis using a distributed lag non-linear model (DLNM) to investigate the association between maximum daily temperature and NCD-YLL.

A natural cubic spline with $8 \mathrm{df}$ per year was applied to control for season and long-term time trends. A heaping effect was found in the raw data (see online supplementary tables 3-5 and supplementary figures 1 and 2), where deaths of an unknown date were assigned to the ninth day of the corresponding month. An indicator variable was added to mark and control for heaping of deaths and day of the week. Exploratory analyses are found in online supplementary figures 3-5. The DLNM captured the immediate and delayed effects of temperature (lags) on health, known as the lag-response association as single lag days, or as it cumulates over time. The exposureresponse curve was modelled with a natural cubic spline with knots placed at the 10th, 50th and 90th percentiles. The lag-response was modelled with a natural cubic

Table 1 Cause-specific NCD outcomes with corresponding deaths and years of life lost. NCD accounted for $12 \%$ of total deaths and $7 \%$ of total YLL in Nouna between 2000 and 2010

\begin{tabular}{lcccc}
\hline Disease & Death count & Death (\%) & YLL count & YLL (\%) \\
\hline Cardiovascular diseases & 461 & 58 & 9095 & 50 \\
Digestive diseases & 137 & 17 & 3614 & 20 \\
Malignant neoplasms & 81 & 10 & 1720 & 9 \\
Genitourinary diseases & 38 & 5 & 1602 & 9 \\
Neuropsychiatric conditions & 37 & 5 & 1289 & 7 \\
Congenital anomalies & 8 & 1 & 481 & 3 \\
Respiratory diseases & 15 & 2 & 321 & 2 \\
Diabetes mellitus & 11 & 1 & 167 & 1 \\
Other endocrine disorders & 1 & 0 & 22 & 0 \\
Musculoskeletal diseases & 1 & 0 & 57 & 0 \\
\hline Total & 790 & 100 & 18367 & 100 \\
\hline
\end{tabular}

NCD, non-communicable disease; YLL, years of life lost. 
Table 2 Summary statistics of daily NCD deaths, NCD years of life lost and temperature in Nouna, Burkina Faso, between 2000 and 2010

\begin{tabular}{|c|c|c|c|c|c|c|}
\hline & \multicolumn{6}{|c|}{ Daily NCD and temperature descriptive statistics $2000-2010$} \\
\hline & Minimum & $25 \%$ & $50 \%$ & Mean & $75 \%$ & Maximum \\
\hline \multicolumn{7}{|c|}{ Daily number of NCD deaths } \\
\hline Total & 0 & 0 & 0 & 0.2 & 0 & 5 \\
\hline Male & 0 & 0 & 0 & 0.1 & 0 & 4 \\
\hline Female & 0 & 0 & 0 & 0.1 & 0 & 3 \\
\hline$>65$ years & 0 & 0 & 0 & 0.1 & 0 & 3 \\
\hline \multicolumn{7}{|c|}{ Daily NCD years of life lost } \\
\hline Total & 0 & 0 & 0 & 4.6 & 0 & 154 \\
\hline Male & 0 & 0 & 0 & 2.4 & 0 & 118.9 \\
\hline Female & 0 & 0 & 0 & 2.1 & 0 & 127.5 \\
\hline$>65$ years & 0 & 0 & 0 & 0.9 & 0 & 39.2 \\
\hline \multicolumn{7}{|l|}{ Temperature $\left({ }^{\circ} \mathrm{C}\right)$} \\
\hline Daily minimum & 3.3 & 21.1 & 22.8 & 23.1 & 25 & 32.8 \\
\hline Daily average & 17.2 & 27.5 & 29.2 & 29.6 & 31.7 & 37.8 \\
\hline Daily maximum & 22.8 & 33.3 & 36.4 & 36.1 & 38.9 & 43.9 \\
\hline
\end{tabular}

NCD, non-communicable disease.

spline of $2 \mathrm{df}$, resulting in default knot placement equally along a logarithmic scale. The model equation was:

$$
\begin{gathered}
E\left(Y_{t}\right)=\beta_{o}+s(T, \text { timed } f)+f\left(X_{\text {tmax }}, \text { lagd } f, \text { vard } f\right)+D O W+H P \\
E\left(Y_{t}\right) \sim \text { quasi }- \text { Poisson }
\end{gathered}
$$

where:

$E\left(Y_{t}\right)$ is the daily YLL,

$\beta_{o}$ is the y intercept,

$s(T$, timedf $)$ is the smooth function of time with specified df timedf

$f\left(X_{\text {tmax }}\right.$, lagdf, vardf $)$ is the cross-basis function of t-max and the associated lag dimension with vardf and $\operatorname{lag} d f \mathrm{df}$, respectively. DOW accounts for day of the week and HP for the heaping effect.

From the RR, absolute values of excess mean daily NCD-YLL were calculated as:

$($ Average daily NCD $-Y L L \times R R)-$ Average daily NCD $-Y L L$

All effect estimates were presented against the median $\mathrm{t}$-max of $36.4^{\circ} \mathrm{C}$ either as overall 4-day and 14-day cumulative RRs (and corresponding excess mean daily NCD-YLL), or single-day lags extending to 14 days.

Several sensitivity analyses were conducted to test the robustness of altering model choices, including: specifying alternative knot positions for exposure-response at the 10th, 75th and 90th, and 10th, 25th, 75th and 90th percentiles, extending df for the lag-response between 2 and $6 \mathrm{df}$, manipulating control for season and time trend ranging from 5 to $10 \mathrm{df}$, logarithm transformation of YLL and applying a Gaussian distribution, and extending the lag period to 28 days to assess if temperature exposure triggered NCD deaths on a longer time scale. QuasiAkaike information criteria (QAIC) values were calculated to guide model selection. All statistical analyses were conducted using R software V.3.2.2. DLNMs were fitted using the DLNM package V.2.2.3.
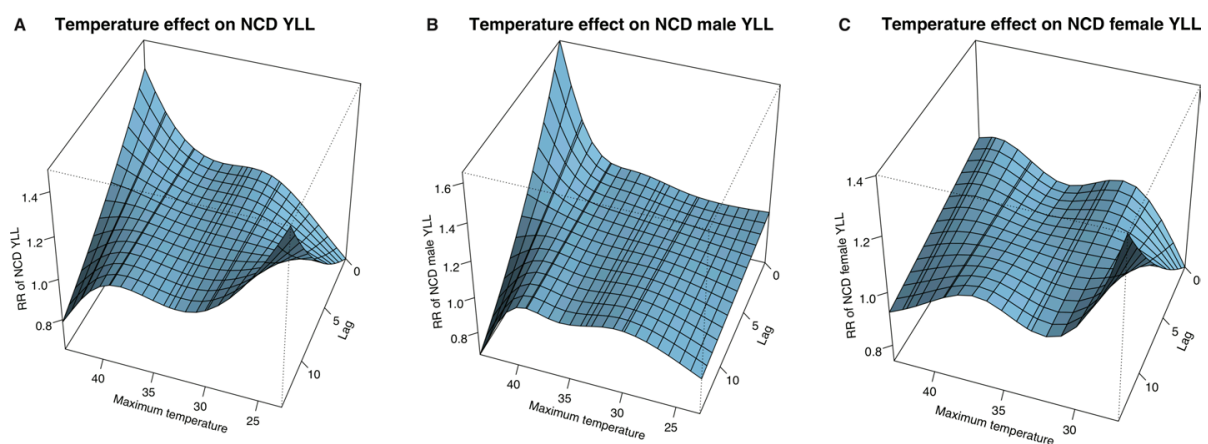

Figure 1 Association of RR of NCD-YLL to maximum temperature and lag days, with reference to $36.4^{\circ} \mathrm{C}$ for $(A)$ all ages, (B) men and (C) women. NCD, non-communicable disease; RR, relative risk; YLL, years of life lost. 
contributor to NCD-YLL, accounting for 9095 or $50 \%$ of all NCD-YLL. Digestive disorders, malignant neoplasms, and genitourinary and neuropsychiatric conditions also contributed substantially towards NCD-YLL. Interestingly, endocrine disorders (including diabetes mellitus) formed a very small proportion (1\%) of all NCD-YLL (table 1). Table 2 shows that maximum mortality peaked at five deaths per day, corresponding to 154 daily NCD-YLL. Daily mean NCD-YLL were 4.6, 2.4 and 2.1 person-years for all ages, men and women, respectively. Maximum daily temperature was $36.4^{\circ} \mathrm{C}$ at the 50th percentile, peaking at $43.9^{\circ} \mathrm{C}$ in the study period.

Figure 1 shows 3D graphs of the RR of NCD-YLL at a range of maximum temperature and lag values, centred at the reference temperature of $36.4^{\circ} \mathrm{C}$ (all RRs and excess mean daily NCD-YLL in the Results section are given as a comparison to this reference temperature). All-age (panel A) and male (panel B) plots showed a strong surge in the RR with high temperature close to the time of heat exposure. Men presented no noticeable effect with colder temperature. In contrast, women (panel $\mathrm{C}$ ) and the all-age group showed more prominent health effects with cooler temperatures, which increased at longer lags. The lag structure of 0-4 days was used to identify immediate health effects, ${ }^{22}$ which were expanded to 14 days to verify if the effects persisted or were concentrated in earlier days. Single-day lagged effects from 0 to 14 days were also considered to identify mortality displacement trends with longer lags. The main results were the 4-day and 14-day cumulative (table 3 and figure 2) and single-day lagged RR of NCD-YLL (table 4 and figure 3), from which daily excess mean NCD-YLL were calculated (tables 5 and 6 ).

Heat effects on NCD-YLL were felt strongly in Nouna above the 50th percentile. Over 4 cumulative days, exposure to moderate temperature (90th percentile at $41.3^{\circ} \mathrm{C}$ ) was associated with a statistically significant increase in excess mean daily NCD-YLL by 4.44 (95\% CI 0.24 to 12.28$)$ for all ages, 3.73 (95\% CI 0.33 to 11.39$)$ for men, but remained statistically insignificant for women, 0.43 (95\% CI -1.08 to 4.16$)$. In comparison to the 90th percentile, excess mean daily NCD-YLL increased slightly at 95 th percentile $\left(41.7^{\circ} \mathrm{C}\right)$ for all ages and men, but not women (table 4). Extreme heat exposure (99th percentile) over 4 days increased excess daily mean NCD-YLL for all ages to 7.39 (95\% CI 0.32 to 24.62$)$ and 8.65 (95\% CI 1.07 to 32.73 ) for men in contrast to the minimal increase for women; 0.12 (95\% CI -1.48 to 5.86). Extending the cumulative effect to 14 days also resulted in elevated excess daily mean NCD-YLL, but wider 95\% confidence bounds rendered the effect estimates for all three groups statistically insignificant. Detailed plots of cumulative effects are found in online supplementary figures 8-10.

Across 14 individual lag days (figure 3), the largest heat effects were felt immediately (at lag 0 ); excess daily mean NCD-YLL were 0.81 (95\% CI 0.13 to 1.59) for all ages, 0.58 (95\% CI 0.11 to 1.15 ) for men, and 0.15 (95\% CI -0.25 to 0.63 ) for women at $41.7^{\circ} \mathrm{C}$ (table 6). Heat effects tapered after lag 0 , but remained statistically significant to

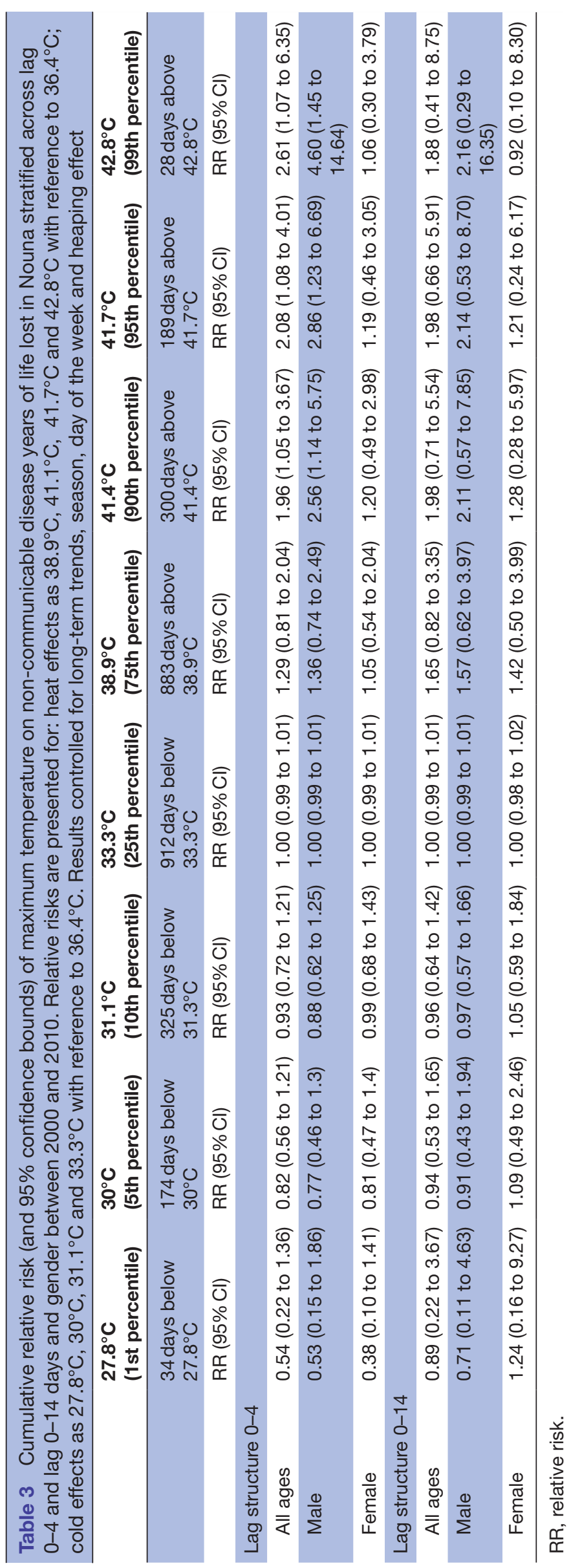



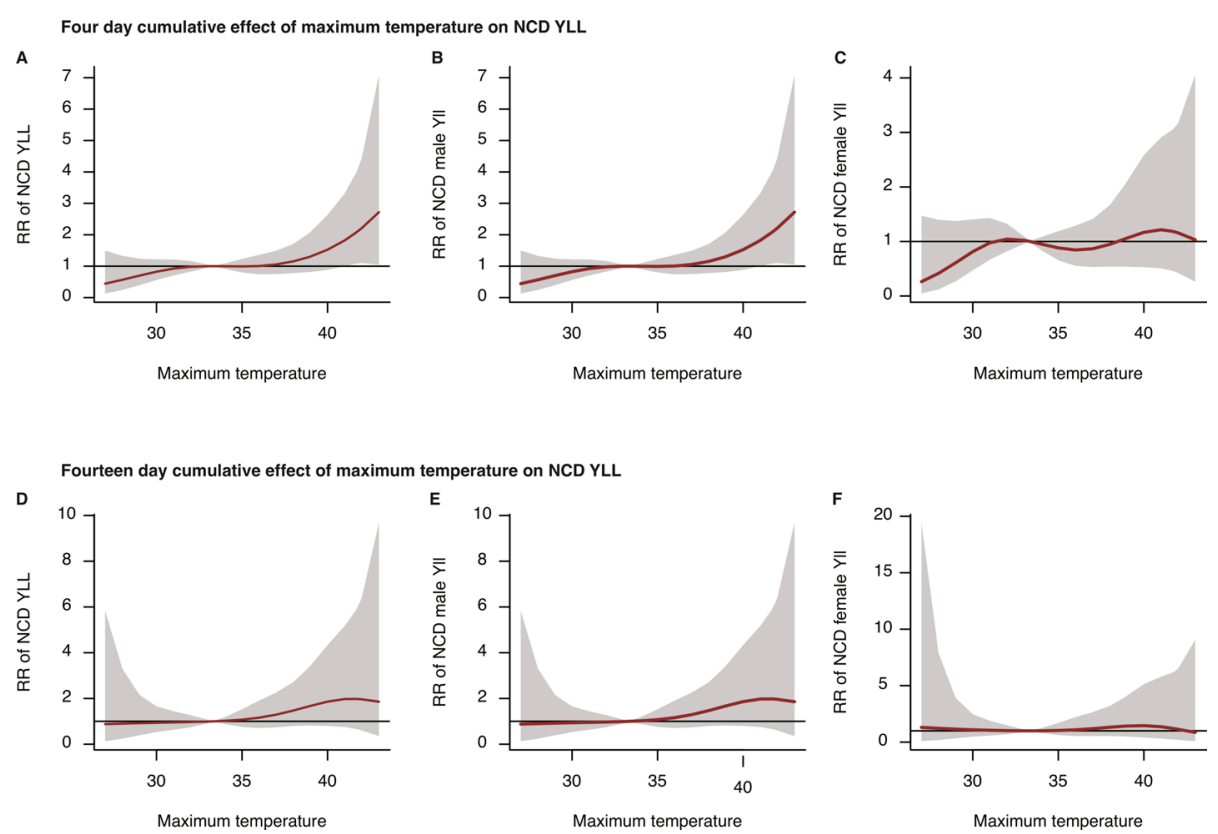

Figure 2 Plots of 4-day (A: all ages, B: male, C: female) and 14-day (D: all ages, E: male, F: female) cumulative RR of NCDYLL against maximum temperature (solid line) with 95\% confidence bounds (grey area) for all ages, men and women in Nouna, Burkina Faso, between 2000 and 2010. The reference temperature is $36.4^{\circ} \mathrm{C}$. Note: To improve legibility of the curves, the scales on the y-axis differ. NCD, non-communicable disease; RR, relative risk; YLL, years of life lost.

lag 4 at $41.7^{\circ} \mathrm{C}$ for all ages and men. For the 95 th percentile, a gradual reduction in excess daily mean NCD-YLL (statistically insignificant) was observed up to 8-10 lag days for all ages, men and women, with no subsequent increase. Detailed risk estimates for individual lag days are found in online supplementary tables $6-8$.

A reduction in temperature to $30^{\circ} \mathrm{C}$ (figure 3 ) resulted in a slightly protective effect at shorter lags $(0-5)$, but after 14 days the excess daily NCD-YLL were slightly elevated for all subgroups: $0.13(95 \%$ CI -0.21 to 0.49$)$ for all ages; 0.06 (95\% CI -0.17 to 0.32$)$ for men; and 0.11 (95\% CI -0.11 to 0.34 ) for women (table 6 ). Women were the only group to present a statistically significant increase in mean daily NCD-YLL with extreme cold (first percentile) at lags 13 and 14 (see online supplementary table $8 b)$.

Excess mean daily NCD-YLL were elevated with heat exposure for the 65+ age group; however, the low sample size produced very large confidence bounds (ie, 0.14 $(95 \% \mathrm{CI}-0.89$ to 86.35$)$ ) at $38.9^{\circ} \mathrm{C}$ vs $36.4^{\circ} \mathrm{C}$.

Several sensitivity analyses were conducted to validate model selection, including generating QAIC, where lower QAIC indicate better model fit (see online supplementary tables 9 and 10). Because increasing the $\mathrm{df}$ produces lower QAIC values, we used prior examples to achieve a balance in controlling for season and long-term trend to $8 \mathrm{df}$ per year. ${ }^{9}$ Applying 7 df per year, as used in other studies, did not greatly affect the risk estimates. ${ }^{21}{ }^{23}$ The natural cubic spline produced lower QAIC in comparison to the more flexible cubic B-spline. Varying knot position and numbers for the exposure-response relationship also did not vary effect estimates. Using $3 \mathrm{df}$ for the lag-response relationship produced the classic reversed J curve expected for heat effects; however, 2 df generated lower QAIC indicating better model fit. There was no evidence of autocorrelation (see online supplementary figures 6 and 7).

\section{DISCUSSION}

A central finding of this study was that excess premature deaths from NCD increased with moderate and extreme heat in rural Sub-Saharan Africa. The magnitude of health effects worsened with heat intensity. The largest increase in excess premature mortality from NCD occurred rapidly on the day of heat exposure (lag 0 ), and diminished in statistical significance after 4 days. The effects of heat on NCD-YLL were greater in men in comparison to women.

In Nairobi, Kenya, increase in temperature over 14 days from $26^{\circ} \mathrm{C}$ to $30^{\circ} \mathrm{C}$ resulted in $3.3(95 \% \mathrm{CI}-19.7$ to 26.4) YLL per day, but from all causes. ${ }^{12}$ Similarly, a change in temperature from the 50th to 75th percentile $\left(36.4^{\circ} \mathrm{C}-38.9^{\circ} \mathrm{C}\right)$ in Nouna resulted in $3.01(95 \%$ CI -0.84 to 10.82 ) excess daily NCD-YLL over 14 days. Unlike Nouna, the temperature in Nairobi does not typically exceed $40^{\circ} \mathrm{C}$. As the only existing African study presenting outcomes as YLL, the comparison presented here indicated $\sim 3$ daily YLL in Nouna and Nairobi with a similar temperature increase. Unfortunately, a direct contrast of results between these two African studies is limited because YLL in Nouna were from NCD only, but from all causes of death in Nairobi.

In Australia and China, heat exposure increased the YLL from cardiovascular disease. A total of 45 years were lost daily from cardiovascular disease (95\% CI 22 to 67 


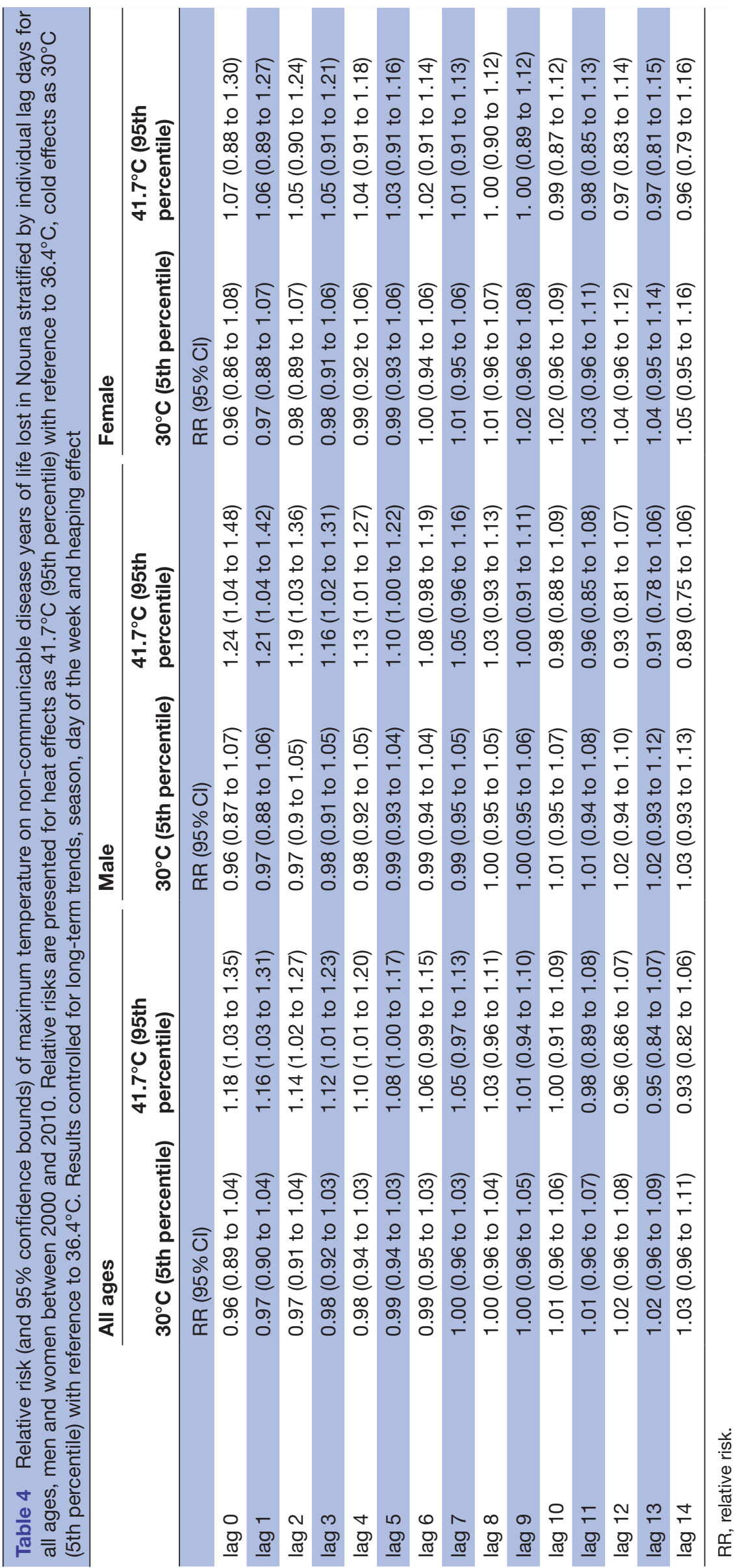



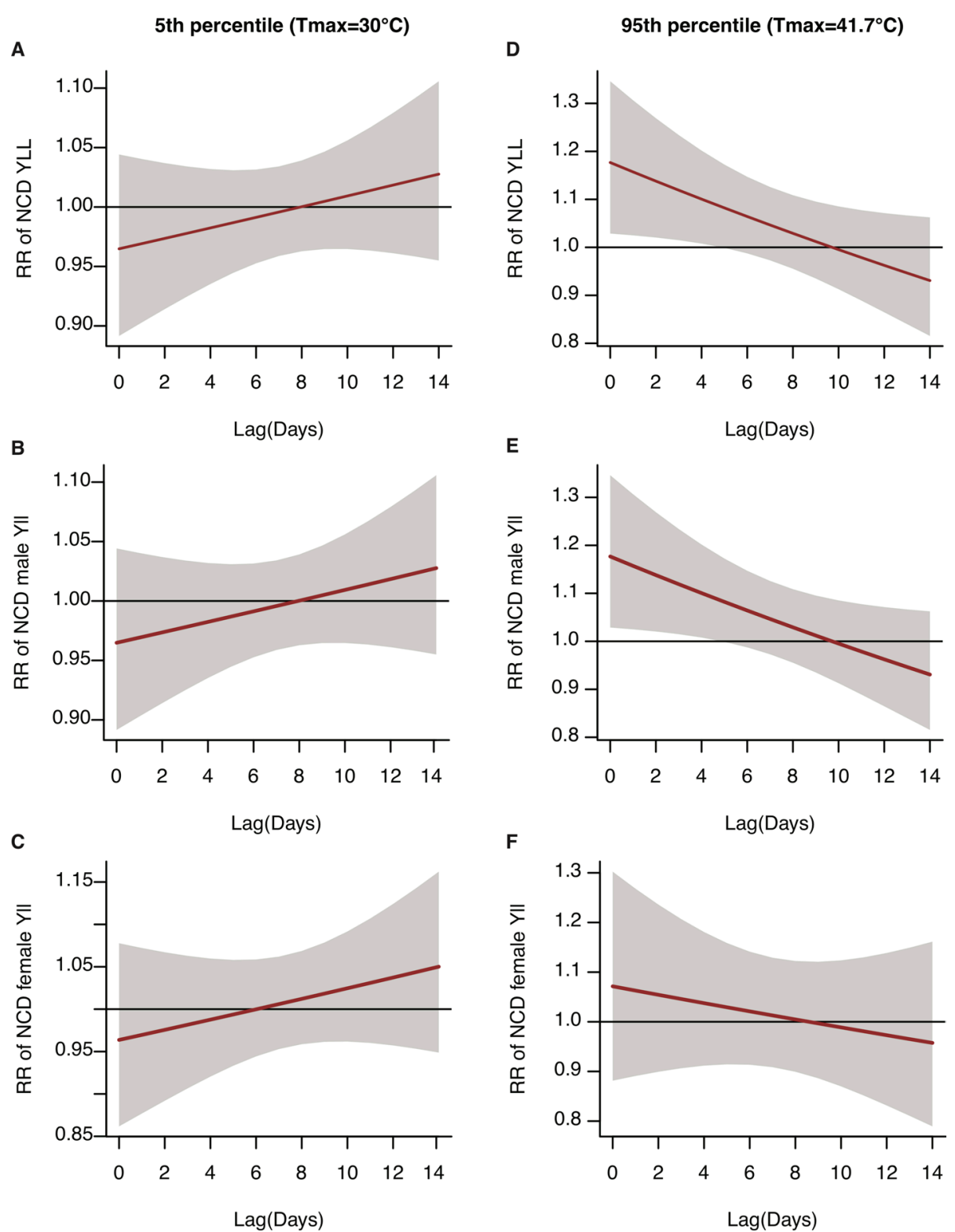

Figure 3 Delayed effects of maximum temperature on the RR of NCD-YLL (solid line) and 95\% confidence bounds (grey area) for all ages, men and women in Nouna, Burkina Faso, by lag 0-14 days. Plots (A) all ages, (B) male and (C) female represent cold effects at $30^{\circ} \mathrm{C}\left(5\right.$ th percentile), and plots (D) all ages, (E) male and (F) female represent heat effects at $41.7^{\circ} \mathrm{C}(95$ th percentile) of maximum temperature. The reference temperature is $36.4^{\circ} \mathrm{C}$. Note: To improve legibility of the curves, the scales on the y-axis differ. NCD, non-communicable disease; RR, relative risk; YLL, years of life lost.

years) in Brisbane, Australia, at a mean temperature of $32^{\circ} \mathrm{C}$ vs $24^{\circ} \mathrm{C}^{19}$ In Guangzhou, China (lags 0-14), a change in mean temperature from the 75 th percentile $\left(28^{\circ} \mathrm{C}\right)$ to the 99 th percentile $\left(32^{\circ} \mathrm{C}\right)$ resulted in 4.81 (95\% CI -2.25 to 11.88$)$ daily YLL from cardiovascular disease. ${ }^{21}$ Cardiovascular disease contributed to $50 \%$ of YLL in Nouna. Although subgroup analysis of NCD was limited by sample size in Nouna, the magnitude of effects was closer to Guangzhou than Brisbane; 4.07 (95\% CI -2.73 to 35.66$)$ and 7.39 (95\% CI 0.32 to 24.62$)$ mean daily YLL were found from all NCDs at lags 0-14 and lags $0-4$, respectively, at the 50th vs 99th percentile. Heat can exacerbate cardiovascular strain through increased cardiac output, blood viscosity and coagulation, attenuated vasoconstriction and cerebral perfusion pressure. ${ }^{24}$ Our findings agree with those from Guangzhou and Brisbane, ${ }^{19} 21$ where heat effects occurred rapidly at lag 0 , lasting a maximum of 4 days. In contrast to Brisbane, Nouna and Guangzhou exhibited fewer YLL for a similar age and temperature shift. All sites used regional or local life tables to calculate YLL rather than global life tables, so the elevated YLL in Brisbane are unlikely to be attributable to lower life expectancy in Nouna compared with Brisbane. Unlike Brisbane, the predominant cause of death in Nouna is still infectious disease; most days in the Nouna time series exhibited no YLL from NCD. Temperature-related premature death from NCD could increase in Nouna as the epidemiological transition progresses, 


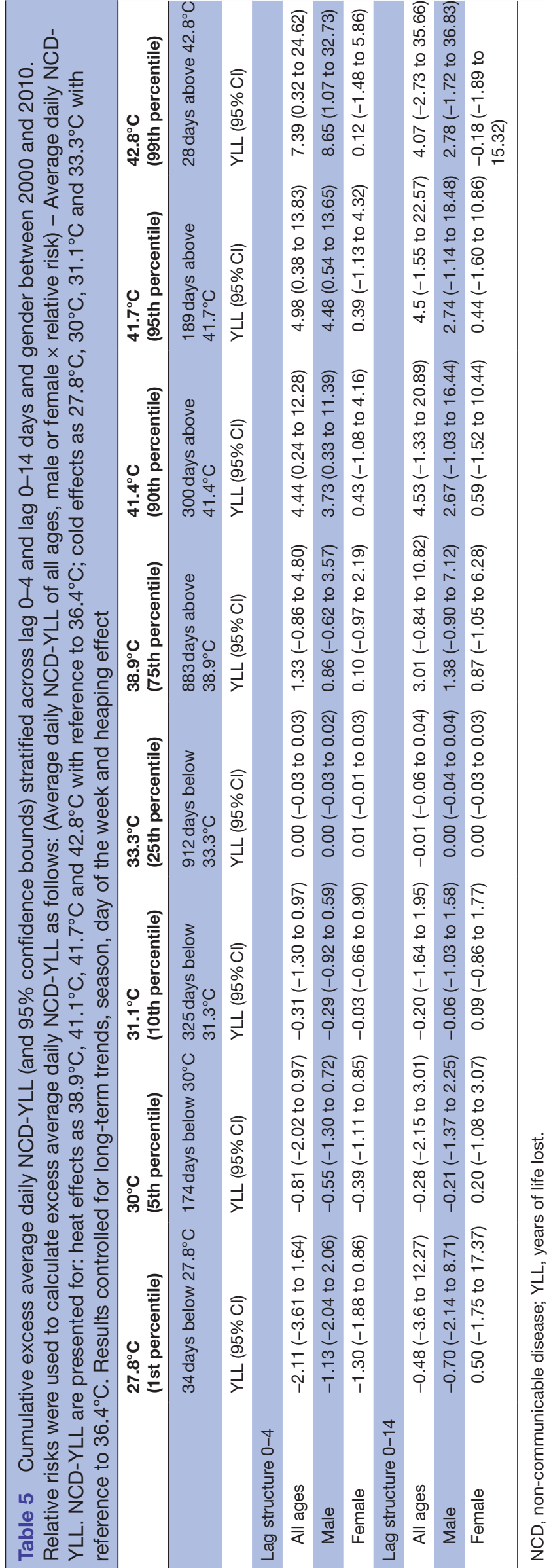

increasing the proportion of deaths attributable to NCD in the future.

Daily respiratory YLL increased by $2.81(95 \%$ CI -1.54 to 7.16) in Guangzhou at $28^{\circ} \mathrm{C}$ versus $32^{\circ} \mathrm{C}^{21}$ where infectious and chronic respiratory deaths were grouped together as ICD-10 J00-99. In the current analysis, however, chronic respiratory YLL (ICD-10 J30-98) only contributed to $2 \%$ of total NCD-YLL in Nouna. The separation of chronic and acute respiratory outcomes may be relevant for comparing findings from different studies and understanding the causal mechanisms. Digestive, renal and neuropsychiatric causes contributed substantially to overall NCD-YLL in Nouna. Heat is known to trigger renal $^{25}$ and mental health-related deaths ${ }^{26}$; however, the link to chronic digestive causes requires further investigation.

Although excess NCD-YLL for women were elevated with heat exposure, male NCD-YLL were affected by a greater magnitude at lags $0-2$. One explanation is that men working outdoors might have higher exposure to ambient heat. Occupational stress has been associated with excess risk of NCD morbidity including psychological distress ${ }^{27}$ and kidney disease. ${ }^{28}$ These results are somewhat unexpected considering that women in Nouna are exposed to extra heat from cooking and carrying wood/ water for 2-3 hours daily. Women might die prematurely from other causes such as childbirth, leaving men to be more affected by diseases associated with longevity such as cardiovascular disease; however, further investigation of gender differences is warranted.

Contrary to findings across 14 European cities, ${ }^{29}$ we found no evidence of harvesting effects with heat; gradual reduction in YLL across lag days ensuing the initial surge was not associated with significant subsequent negative associations or a rise again in risk estimates for any subgroups. ${ }^{22}$ The public health relevance of our findings is therefore enhanced, as premature NCD mortality is not merely the advancement of death in frail individuals with pre-existing chronic conditions.

This study has several strengths. An 11-year time series of reliable, high-quality data from a rural African setting was used to quantify the burden of temperature on NCD-YLL. Variables such as the date, age and cause of death were subject to quality checks and continuous improvements at INDEPTH (International Network for the Demographic Evaluation of Populations and Their Health) sites including Nouna. These processes enabled one of the best quality and most extensive longitudinal health data sets in Africa and Asia to be used for this study. The DLNM accounted for non-linearity and lagged effects. In place of RRs which would have been obtained had only death counts been used, combining life expectancy and death counts gave an absolute value for YLL from NCD, which is relevant for policymaking. ${ }^{30}$ Despite the low number of NCD deaths, significant effects of heat on premature mortality were detected, indicating that the effects were strong. The results in the final model were robust and withstood variations of model parameters. 


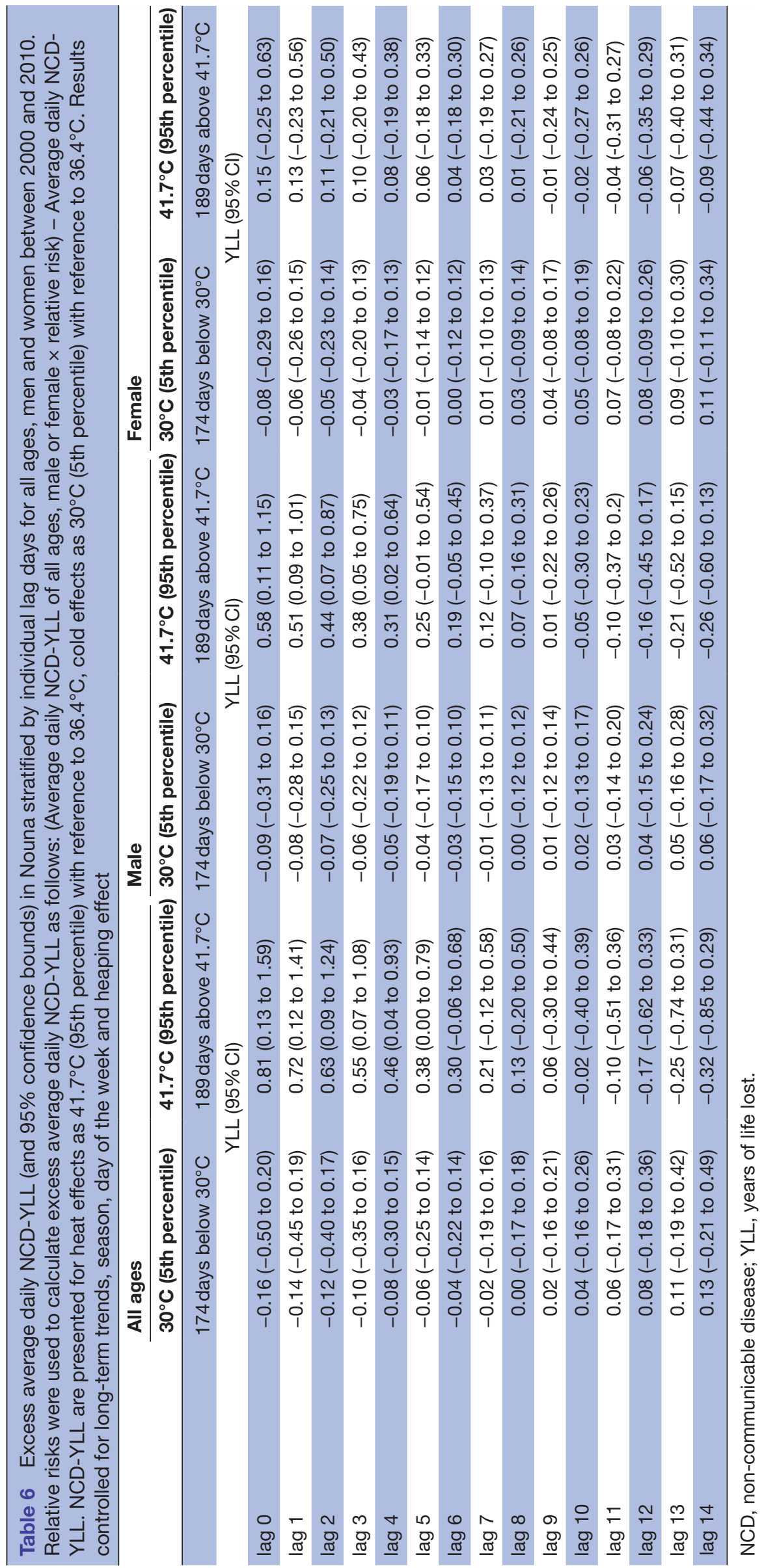


Rather than focusing only on anomalous weather events such as heatwaves, one of the longest time series available in rural Africa was exploited to highlight that excess premature deaths from NCD occur during extreme heat and with moderate heat.

Some limitations are also noted. Caution should be exercised in generalising these findings to all rural African settings. Temperature data were obtained from the nearest location with a similar temperature profile to Nouna. Air pollution data were unavailable to assess potential confounding effects of the exposure-response relationship. The lower resolution and distribution of weather data in Burkina Faso compared with Organisation for Economic Co-operation and Development countries can make it challenging to obtain suitable weather data in Burkina Faso. Public health scientists ought to address this challenge by extending research beyond where the data are best, to where problems are the greatest and research/solutions most needed. It is likely that cancer or mental disorders were under-reported as sophisticated questionnaires and tests are needed to establish these causes. In 2004, the WHO estimated that NCD accounted for $20 \%$ of the burden of disease in Burkina Faso as a percentage of total disability-adjusted life years (DALY), which captures both premature death and life lived with disease. ${ }^{31}$ We found that only $7 \%$ of the burden from premature deaths or YLL in Nouna were from NCD. Although the YLL component of DALYs in the WHO estimate was obtained by multiplying the number of deaths at each age by the global standard life expectancy for each age (rather than the regional life expectancy for each age), the sole use of premature death is likely to have missed substantial burden from life lived with disease. Causal studies on the temperature-NCD association would benefit from using DALYs or quality-adjusted life years as the outcome measure, considering a large proportion of the burden of NCD comes from life lived with disease. The YLL life table approach does not differentiate health and sociodemographic risk profiles for each individual. Unfortunately, the sample size was insufficient to further stratify NCD by age (ie, elderly) or subgroups such as cardiovascular causes. The use of longer time series in the future with larger sample sizes is likely to enable such breakdowns by cause or age, reducing the uncertainty from wide confidence bounds, and supporting better quantification of heat impacts on NCD-YLL.

\section{CONCLUSION}

In rural Sub-Saharan Africa, where NCDs are not the main cause of premature death, we found that moderate and extreme heat exposure significantly increases excess daily premature mortality from NCD. As NCD prevalence increases in Africa due to demographic, dietary and lifestyle changes, climate change will increasingly contribute as a risk factor towards the burden of deaths from NCD. Subsistence farming communities in Africa, such as
Nouna, would therefore benefit from the development of early preventive measures to curb heat-associated NCD deaths.

Correction notice This article has been corrected since it first published. The Acknowledgements have been added in.

Acknowledgements We thank Dr David Hondula for help obtaining temperature data from the National Climatic Data Centre, USA, and Drs Adrian Barnett and Cunrui Huang for statistical modelling advice.

Contributors $A B$ and $R S$ developed the research idea with input from JR. Data were provided by $A S$. $A B, M O S, J R$ and RS developed the modelling strategy. $A B$ conducted the analysis, which was verified by MOS and JR. $A B$ wrote the manuscript. All authors contributed to revision of the manuscript.

Funding $A B$ was funded by the Klaus-Tschira Stiftung $\mathrm{gGmbH}(00.128 .2008)$. Competing interests None declared.

Provenance and peer review Not commissioned; externally peer reviewed. Data sharing statement There is no additional unpublished work.

Open Access This is an Open Access article distributed in accordance with the Creative Commons Attribution Non Commercial (CC BY-NC 4.0) license, which permits others to distribute, remix, adapt, build upon this work non-commercially, and license their derivative works on different terms, provided the original work is properly cited and the use is non-commercial. See: http://creativecommons.org/ licenses/by-nc/4.0/

(c) Article author(s) (or their employer(s) unless otherwise stated in the text of the article) 2017. All rights reserved. No commercial use is permitted unless otherwise expressly granted.

\section{REFERENCES}

1 Niang I, Ruppel OC, Abdrabo MA, et al. Africa. In: climate change 2014: impacts, adaptation, and vulnerability-part B: regional aspectscontribution of working group II to the fifth assessment report of the intergovernmental panel on climate change. NY, USA: Cambridge University Press, 2014.

2. Hondula DM, Rocklöv J, Sankoh OA. Past, present, and future climate at select INDEPTH member Health and Demographic Surveillance Systems in Africa and Asia. Glob Health Action 2012;5:19083-6.

3. Abegunde DO, Mathers CD, Adam T, et al. The burden and costs of chronic diseases in low-income and middle-income countries. Lancet 2007;370:1929-38.

4. World Health OrganizationBurkina Faso. Non-communicable Diseases (NCD) country profiles. 2014 http://www.who.int/nmh/ countries/bfa_en.pdf (accessed 12 May 2016).

5. Friel S, Bowen K, Campbell-Lendrum D, et al. Climate change, noncommunicable diseases, and development: the relationships and common policy opportunities. Annu Rev Public Health 2011;32:133-47.

6. D'Ippoliti D, Michelozzi P, Marino C, et al. The impact of heat waves on mortality in 9 European cities: results from the EuroHEAT project. Environ Health 2010;9:37.

7. Fouillet A, Rey G, Laurent F, et al. Excess mortality related to the August 2003 heat wave in France. Int Arch Occup Environ Health 2006;80:16-24.

8. IPCC. Summary for Policymakers. Climate change 2013: the physical science basis. contribution of working group I to the fifth assessment report of the intergovernmental panel on climate change. United Kingdom and New York, NY, USA: Cambridge University Press, Cambridge.

9. Gasparrini A, Guo Y, Hashizume M, et al. Mortality risk attributable to high and low ambient temperature: a multicountry observational study. Lancet 2015;386:369-75.

10. Diboulo E, Sié A, Rocklöv J, et al. Weather and mortality: a 10 year retrospective analysis of the Nouna Health and Demographic Surveillance System, Burkina Faso. Glob Health Action 2012;5:19078-8.

11. Egondi $T$, Kyobutungi $C$, Kovats $S$, et al. Time-series analysis of weather and mortality patterns in Nairobi's informal settlements. Glob Health Action 2012;5:1-10.

12. Egondi T, Kyobutungi C, Rocklöv J. Temperature variation and heat wave and cold spell impacts on years of life lost among the urban 
poor population of Nairobi, Kenya. Int J Environ Res Public Health 2015;12:2735-48.

13. Sié A, Louis VR, Gbangou A, et al. The Health and Demographic Surveillance System (HDSS) in Nouna, Burkina Faso, 1993-2007. Glob Health Action 2010;3.

14. Ramroth $\mathrm{H}$, Lorenz E, Rankin JC, et al. Cause of death distribution with InterVA and physician coding in a rural area of Burkina Faso. Trop Med Int Health 2012;17:904-13.

15. World Health Organization. The global burden of disease. Geneva 2008. updated 2004

16. Murray CJ, Lopez AD, eds. The global burden of disease: a comprehensive assessment of mortality and disability from diseases, injuries, and risk factors in 1990 and projected to 2020. Geneva: World Health Organization, 1996.

17. In: Pruss-Ustun A, Mathers C, Corvolan C, eds. Introduction and methods: assessing the environmental burden of disease at national and local levels. Geneva, 2003.

18. Murray CJ, Ezzati M, Flaxman AD, et al. GBD 2010: design, definitions, and metrics. Lancet 2012;380:2063-6.

19. Huang C, Barnett AG, Wang X, et al. Effects of extreme temperatures on years of life lost for cardiovascular deaths: a time series study in Brisbane, Australia. Circ Cardiovasc Qual Outcomes 2012;5:609-14.

20. Huang $C$, Barnett AG, Wang $X$, et al. The impact of temperature on years of life lost in Brisbane, Australia. Nat Clim Chang 2012;2:265-70.

21. Yang J, Ou CQ, Guo Y, et al. The burden of ambient temperature on years of life lost in Guangzhou, China. Sci Rep 2015;5:12250.

22. Basu R, Malig B. High ambient temperature and mortality in California: exploring the roles of age, disease, and mortality displacement. Environ Res 2011;111:1286-92.
23. Bhaskaran K, Gasparrini A, Hajat S, et al. Time series regression studies in environmental epidemiology. Int $J$ Epidemiol 2013;42:1187-95.

24. Keatinge WR, Coleshaw SR, Easton JC, et al. Increased platelet and red cell counts, blood viscosity, and plasma cholesterol levels during heat stress, and mortality from coronary and cerebral thrombosis. Am J Med 1986;81:795-800.

25. Semenza JC, McCullough JE, Flanders WD, et al. Excess hospital admissions during the July 1995 heat wave in Chicago. Am J Prev Med 1999;16:269-77.

26. Basu R, Samet JM. The potential impacts of climate variability and change on temperature-related morbidity and mortality in the United States. Environ Health Perspect 2002;110:1219-24.

27. Tawatsupa B, Lim LL, Kjellstrom T, et al. The association between overall health, psychological distress, and occupational heat stress among a large national cohort of 40,913 Thai workers. Glob Health Action 2010;3:5034

28. Tawatsupa B, Lim LL, Kjellstrom T, et al. Association between occupational heat stress and kidney disease among 37,816 workers in the Thai Cohort Study (TCS). J Epidemiol 2012;22:251-60.

29. Baccini M, Kosatsky T, Biggeri A. Impact of summer heat on urban population mortality in Europe during the 1990s: an evaluation of years of life lost adjusted for harvesting. PLoS One 2013;8:e69638.

30. Murray CJ, Lopez AD. Measuring the global burden of disease. $N$ Engl J Med 2013;369:448-57.

31. World Health Organization Regional Office for Africa. Health situation analysis in the African region. Brazzaville 2011. 